\title{
Symplectic difference systems with periodic coefficients: Krein's traffic rules for multipliers
}

\section{Ondřej Došlý}

\section{${ }^{*}$ Correspondence:}

dosly@math.muni.cz

Department of Mathematics,

Masaryk University, Kotlárská 2,

Brno, CZ-611 37, Czech Republic

\begin{abstract}
We investigate symplectic difference systems with periodic coefficients. We show that the known traffic rules for eigenvalues of the monodromy matrix of linear Hamiltonian differential and difference systems can be extended also to discrete symplectic systems.
\end{abstract}

MSC: 39A12; 15A18

Keywords: symplectic difference system; periodic; traffic rules; monodromy matrix; definite multiplier

\section{Introduction}

A symplectic difference system is the discrete first-order system

$$
z_{k+1}=\mathcal{S}_{k} z_{k}, \quad k \in \mathbb{Z},
$$

where the matrices $\mathcal{S}_{k} \in \mathbb{C}^{2 n \times 2 n}$ are symplectic, i.e.,

$$
\mathcal{S}_{k}^{*} \mathcal{J} \mathcal{S}_{k}=\mathcal{J}, \quad \mathcal{J}=\left(\begin{array}{cc}
0 & I \\
-I & 0
\end{array}\right) .
$$

In particular, if $\mathcal{S}_{k} \in \mathbb{R}^{2 n \times 2 n}$, then we have $\mathcal{S}_{k}^{T} \mathcal{J} \mathcal{S}_{k}=\mathcal{J}$. The terminology for symplectic matrices is not unique. Our terminology is taken from [1]. An alternative terminology $\mathcal{J}$-unitary, $\mathcal{J}$-orthogonal can be found in [2]. Also, complex-valued matrices satisfying (1) are sometimes called complex symplectic.

Symplectic difference systems are discrete counterparts of linear Hamiltonian differential systems

$$
z^{\prime}=\mathcal{J H}(t) z
$$

with the Hermitean matrix $\mathcal{H}$, i.e., $\mathcal{H}^{*}=\mathcal{H}$, in the sense that they are the most general firstorder systems whose fundamental matrix is symplectic. It is known that one can establish oscillation and Sturmian theory for real symplectic difference systems similar to those for Hamiltonian differential systems, even if passing from continuous to discrete is far from being trivial. We refer to $[3,4]$ and the references given therein. Note that symplectic difference systems cover, as a very special case, the second-order Sturm-Liouville difference equation

$$
\Delta\left(r_{k} \Delta x_{k}\right)+c_{k} x_{k+1}=0, \quad r_{k} \neq 0,
$$

@ 2013 Došlý; licensee Springer. This is an Open Access article distributed under the terms of the Creative Commons Attribution License (http://creativecommons.org/licenses/by/2.0), which permits unrestricted use, distribution, and reproduction in any medium, provided the original work is properly cited. 
whose qualitative theory is deeply developed; see, e.g., [5].

In our paper we suppose that the matrices $\mathcal{S}_{k}$ depend on a (generally complex-valued) parameter $\lambda$, i.e., we consider the system

$$
z_{k+1}=\mathcal{S}_{k}(\lambda) z_{k}
$$

Based on the papers [6,7], see also the parts (i), (ii) of the next section, our assumptions on the matrices $\mathcal{S}_{k}(\lambda)$ are following:

(A) System (2) is symplectic for $\lambda \in \mathbb{R}$, i.e.,

$$
\mathcal{S}_{k}^{*}(\lambda) \mathcal{J} \mathcal{S}_{k}(\lambda)=\mathcal{J}, \quad k \in \mathbb{Z}
$$

(B) The matrices $\mathcal{S}_{k}(\lambda)$ are periodic with the period $N \in \mathbb{N}$, i.e.,

$$
\mathcal{S}_{k}(\lambda)=\mathcal{S}_{k+N}(\lambda), \quad k \in \mathbb{Z}, \lambda \in \mathbb{C} .
$$

(C) The matrices $\mathcal{S}_{k}(\lambda), k=0, \ldots, N-1$, are analytic in a neighborhood of $\lambda_{0}=0$, in particular,

$$
\mathcal{S}_{k}(\lambda)=\sum_{j=0}^{\infty} S_{k}^{[j]} \lambda^{j}, \quad S_{k}^{[0]}=I,
$$

where $S_{k}^{[j]} \in \mathbb{C}^{2 n \times 2 n}$ satisfy the periodicity condition

$$
S_{k+N}^{[j]}=S_{k}^{[j]}, \quad k \in \mathbb{Z}, j \in \mathbb{N} .
$$

Our principal concern is to study the stability (for $k \in \mathbb{Z}$ ) of solutions of (2). Concerning the continuous counterpart of symplectic difference systems, linear Hamiltonian differential systems

$$
z^{\prime}=\lambda \mathcal{J H}(t) z
$$

the stability theory of periodic Hamiltonian differential systems is deeply developed since the fifties of the last century. In that period, Russian mathematicians Gelfand, Krein, Lidskii, Yakubovich, Starzhinskii and others [2, 8, 9] published fundamental papers on stability of (3), which were summarized in the book [10].

The requirement that our system is symplectic for real $\lambda$ reads in terms of the coefficients $S_{k}^{[j]}$ as follows. We suppose that the below given formulas hold for all indices $k=0, \ldots, N-$ 1 , so we suppress the index $k$ :

$$
\begin{aligned}
& \left(S^{[1]}\right)^{*} \mathcal{J}+\mathcal{J} S^{[1]}=0, \\
& \left(S^{[2]}\right)^{*} \mathcal{J}+\mathcal{J} S^{[2]}+\left(S^{[1]}\right)^{*} \mathcal{J} S^{[1]}=0, \\
& \vdots \\
& \left(S^{[n]}\right)^{*} \mathcal{J}+\cdots+\left(S^{[n-j]}\right)^{*} \mathcal{J} S^{[j]}+\cdots+\mathcal{J} S^{[n]}=0,
\end{aligned}
$$


The following statement plays the crucial role in the later introduced concept of a symplectic system of positive type.

Proposition 1 Suppose that (4)-(6) hold. Then there exist Hermitean matrices $\mathcal{A}_{k}(\lambda, \bar{\lambda})$ such that for $\lambda \in \mathbb{C}$

$$
\mathcal{S}_{k}^{*}(\lambda) \mathcal{J} \mathcal{S}_{k}(\lambda)=\mathcal{J}+(\bar{\lambda}-\lambda) \mathcal{A}_{k}(\lambda, \bar{\lambda})
$$

Proof Suppressing again the index $k$, using (4),

$$
\begin{aligned}
\mathcal{S}^{*}(\lambda) \mathcal{J} \mathcal{S}(\lambda)= & \left(I+\sum_{j=1}^{\infty} \bar{\lambda}^{j}\left(S^{[j]}\right)^{*}\right) \mathcal{J}\left(I+\sum_{j=1}^{\infty} \lambda^{j} S^{[j]}\right) \\
= & \mathcal{J}+(\bar{\lambda}-\lambda)\left(-\mathcal{J} S^{[1]}\right) \\
& +(\bar{\lambda})^{2}\left(S^{[2]}\right)^{*} \mathcal{J}+\bar{\lambda} \lambda\left(S^{[1]}\right)^{*} \mathcal{J} \mathcal{S}^{[1]}+\lambda^{2} \mathcal{J} S^{[2]}+\cdots \\
& +(\bar{\lambda})^{n}\left(\mathcal{S}^{[n]}\right)^{*} \mathcal{J}+\cdots+(\bar{\lambda})^{n-j} \lambda^{j}\left(\mathcal{S}^{[n-j]}\right)^{*} \mathcal{J} S^{[j]}+\cdots+\lambda^{n} \mathcal{J} \mathcal{S}^{[n]} \\
& +\cdots
\end{aligned}
$$

Concerning the expression (8), using (5), we immediately have

$$
(\bar{\lambda})^{2}\left(S^{[2]}\right)^{*} \mathcal{J}+\bar{\lambda} \lambda\left(S^{[1]}\right)^{*} \mathcal{J} \mathcal{S}^{[1]}+\lambda^{2} \mathcal{J} S^{[2]}=(\bar{\lambda}-\lambda)\left[\bar{\lambda}\left(\mathcal{S}^{[2]}\right)^{*} \mathcal{J}-\lambda \mathcal{J} \mathcal{S}^{[2]}\right]
$$

Generally, concerning expression (9), consider the matrices $X_{1}^{[n]}, \ldots, X_{n-2}^{[n]}, n \geq 3$, given by the formulas

$$
\begin{aligned}
& X_{1}^{[n]}=\left(S^{[n]}\right)^{*} \mathcal{J}+\left(S^{[n-1]}\right)^{*} \mathcal{J} S^{[1]}, \\
& X_{2}^{[n]}=X_{1}^{[n]}+\left(S^{[n-2]}\right)^{*} \mathcal{J} S^{[2]}, \\
& \vdots \\
& X_{n-2}^{[n]}=X_{n-3}^{[n]}+\left(S^{[2]}\right)^{*} \mathcal{J} S^{[n-2]} .
\end{aligned}
$$

Then formula (6) implies that we also have

$$
-X_{n-2}^{[n]}=\left(S^{[1]}\right)^{*} \mathcal{J} S^{[n-1]}+\mathcal{J} S^{[n]} .
$$

Now, by a direct computation, using (10), we have that (9) for $n \geq 3$ equals

$$
(\bar{\lambda}-\lambda)\left[(\bar{\lambda})^{n-1}\left(S^{[n]}\right)^{*} \mathcal{J}+\cdots+(\bar{\lambda})^{n-j-1} \lambda^{j} X_{j}^{[n]}+\cdots-\lambda^{n-1} \mathcal{J} S^{[n]}\right]
$$

i.e., one can factor out the term $(\bar{\lambda}-\lambda)$ from the expression for $\mathcal{S}^{*}(\lambda) \mathcal{J} \mathcal{S}(\lambda)-\mathcal{J}$. It remains to prove that $\mathcal{A}(\lambda, \bar{\lambda})$ is Hermitean. This matrix is given by an infinite series, and the typical summand in this series is given by the expression in brackets in (11). To prove that this 
expression is Hermitean, it suffices to show that $\left(X_{j}^{[n]}\right)^{*}=X_{n-j-1}^{[n]}$. We have, using (6),

$$
\begin{aligned}
\left(X_{j}^{[n]}\right)^{*} & =\left[\left(S^{[n]}\right)^{*} J+\left(S^{[n-1]}\right)^{*} \mathcal{J} S^{[1]}+\cdots+\left(S^{[n-j]}\right)^{*} \mathcal{J} S^{[j]}\right]^{*} \\
& =-\left[\mathcal{J} S^{[n]}+\left(S^{[1]}\right)^{*} \mathcal{J} S^{[n-1]}+\cdots+\left(S^{[j]}\right)^{*} \mathcal{J} S^{[n-j]}\right] \\
& =\left(S^{[n]}\right)^{\prime \prime} \mathcal{J}+\left(S^{[n-1]}\right)^{*} \mathcal{J} S^{[1]}+\cdots+\left(S^{[j+1]}\right)^{* \prime} \mathcal{J} S^{[n-j-1]} \\
& =X_{n-j-1}^{[n]} .
\end{aligned}
$$

Consequently, each term in the infinite series expressing $\mathcal{A}(\lambda, \bar{\lambda})$ is a Hermitean matrix, and hence this matrix is Hermitean as well.

\section{Particular cases}

In this section, we treat special cases of our periodic symplectic difference system as presented in the recent literature.

(i) Second-order matrix difference system.

The results concerning this system were published in [6]. We consider the second-order matrix difference system with $P_{k} \in \mathbb{C}^{n \times n}$

$$
\Delta^{2} x_{k-1}+\lambda^{2} P_{k} x_{k}=0, \quad P_{k}^{*}=P_{k}, \quad P_{k+N}=P_{k} .
$$

In this case, we write the system in the form

$$
\Delta\left(\frac{1}{\lambda} \Delta x_{k-1}\right)+\lambda P_{k} x_{k}=0
$$

If we put $u_{k}=\frac{1}{\lambda} \Delta x_{k-1}$, then $\Delta u_{k}=-\lambda P_{k} x_{k}$, i.e., $u_{k+1}=-\lambda P_{k} x_{k}+u_{k}$ and $x_{k+1}-x_{k}=\lambda u_{k+1}=$ $\lambda\left(-\lambda P_{k} x_{k}+u_{k}\right)$. Consequently,

$$
\left(\begin{array}{l}
x_{k+1} \\
u_{k+1}
\end{array}\right)=\left[I+\left(\begin{array}{cc}
0 & I \\
-P_{k} & 0
\end{array}\right) \lambda+\left(\begin{array}{cc}
-P_{k} & 0 \\
0 & 0
\end{array}\right) \lambda^{2}\right]\left(\begin{array}{l}
x_{k} \\
u_{k}
\end{array}\right) .
$$

Denote by $S^{[1]}$ and $S^{[2]}$ the matrices by $\lambda$ and $\lambda^{2}$, respectively. By a direct computation, taking into account the form of the matrices $S^{[1]}$ and $S^{[2]}$, we obtain for $\lambda \in \mathbb{C}$

$$
\begin{aligned}
S^{*}(\lambda) & \mathcal{J} S(\lambda) \\
= & \mathcal{J}+\bar{\lambda}\left(S^{[1]}\right)^{*} \mathcal{J}+\lambda \mathcal{J} S^{[1]}+(\bar{\lambda})^{2}\left(S^{[2]}\right)^{*} \mathcal{J}+\bar{\lambda} \lambda\left(S^{[1]}\right)^{*} \mathcal{J} S^{[1]}+\lambda^{2} \mathcal{J} S^{[2]} \\
& +(\bar{\lambda})^{2} \lambda\left(S^{[2]}\right)^{*} \mathcal{J} S^{[1]}+\lambda^{2} \bar{\lambda}\left(S^{[1]}\right)^{*} \mathcal{J} S^{[2]}+(\bar{\lambda} \lambda)^{2}\left(S^{[2]}\right)^{*} \mathcal{J} S^{[2]} \\
= & \mathcal{J}+(\bar{\lambda}-\lambda)\left(-\mathcal{J} S^{[1]}\right)+(\bar{\lambda})^{2}\left(\begin{array}{cc}
0 & -P^{*} \\
0 & 0
\end{array}\right)+\lambda \bar{\lambda}\left(\begin{array}{cc}
0 & P^{*} \\
-P & 0
\end{array}\right) \\
& +\lambda^{2}\left(\begin{array}{ll}
0 & 0 \\
P & 0
\end{array}\right)+\left[(\bar{\lambda})^{2} \lambda-\bar{\lambda} \lambda^{2}\right]\left(\begin{array}{cc}
P^{*} P & 0 \\
0 & 0
\end{array}\right) \\
= & \mathcal{J}+(\bar{\lambda}-\lambda)\left(\begin{array}{cc}
P+|\lambda|^{2} P^{*} P & -\bar{\lambda} P \\
-\lambda P & I
\end{array}\right) .
\end{aligned}
$$


Therefore, (13) is really a symplectic system for $\lambda \in \mathbb{R}$, and to verify (5) and (6) with $n=3,4$ is a matter of an easy computation.

(ii) Linear Hamiltonian difference system.

The stability of this system was investigated in [7]; see also [11]. We consider the system

$$
\Delta\left(\begin{array}{l}
x_{k} \\
u_{k}
\end{array}\right)=\lambda \mathcal{J}\left(\begin{array}{cc}
-C_{k} & A_{k} \\
A_{k}^{*} & B_{k}
\end{array}\right)\left(\begin{array}{c}
x_{k+1} \\
u_{k}
\end{array}\right)
$$

with the Hermitean matrices $B, C$, i.e., $B=B^{*}, C=C^{*}$. This system can be written in the form

$$
\left(\begin{array}{l}
x_{k+1} \\
u_{k+1}
\end{array}\right)=\left(\begin{array}{cc}
\left(I-\lambda A_{k}\right)^{-1} & \lambda\left(I-\lambda A_{k}\right)^{-1} B_{k} \\
\lambda C_{k}\left(I-\lambda A_{k}\right)^{-1} & \lambda^{2} C_{k}\left(I-\lambda A_{k}\right)^{-1} B_{k}+I-\lambda A_{k}^{*}
\end{array}\right)\left(\begin{array}{l}
x_{k} \\
u_{k}
\end{array}\right) .
$$

If we denote by $\mathcal{S}(\lambda)$ the matrix in the last system, we have, using the formula ( $I-$ $\left.\lambda A_{k}\right)^{-1}=\sum_{j=0}^{\infty} \lambda^{j} A_{k}^{j}$ (suppressing the index $k$ ),

$$
\mathcal{S}(\lambda)=I+\lambda\left(\begin{array}{cc}
A & B \\
C & -A^{*}
\end{array}\right)+\sum_{j=2}^{\infty} S^{[j]} \lambda^{j}
$$

with the matrices (for $j \geq 2$ )

$$
S^{[j]}=\left(\begin{array}{cc}
A^{j} & A^{j-1} B \\
C A^{j-1} & C A^{j-2} B
\end{array}\right) .
$$

To show that the matrices $S^{[j]}, j \geq 2$, together with the matrix

$$
S^{[1]}=\left(\begin{array}{cc}
A & B \\
C & -A^{*}
\end{array}\right)
$$

satisfy symplecticity condition (6) requires rather tedious computations. Consider two successive terms in (6)

$$
\left(S^{[n-j+1]}\right)^{*} \mathcal{J} S^{[j-1]}+\left(S^{[n-j]}\right)^{*} \mathcal{J} S^{[j]},
$$

and let us compute the block matrix in the left upper corner. By a direct substitution, we obtain the expression

$$
-\left(A^{*}\right)^{n-j-1} C A^{j}+\left(A^{*}\right)^{n-j} C A^{j-1}-\left(A^{*}\right)^{n-j} C A^{j-1}+\left(A^{*}\right)^{n-j+1} C A^{j-2} .
$$

Hence, we immediately see that the second and third term cancel. Moreover, the first one cancels against a term coming from the expression $\left(S^{[n-j+2]}\right)^{*} \mathcal{J} S^{[j-2]}$, and similarly, the last term in (15) also disappears. By the same reasoning, we also show that the remaining three blocks in (6) equal zero. Also, it is shown in [7] that

$$
\mathcal{S}^{\prime \prime}(\lambda) \mathcal{J} \mathcal{S}(\lambda)=\mathcal{J}+(\bar{\lambda}-\lambda) D^{*}(\lambda)\left(\begin{array}{cc}
-C & A^{*} \\
A & B
\end{array}\right) D(\lambda)
$$


where

$$
D(\lambda)=\left(\begin{array}{cc}
(I-\lambda A)^{-1} & \lambda(I-\lambda A)^{-1} B \\
0 & I
\end{array}\right) .
$$

Therefore, we see that Hamiltonian system (14) is a particular case of our symplectic system (2) satisfying (7).

(iii) Exponential system.

By an 'exponential' system we mean the system (2), where

$$
\mathcal{S}_{k}(\lambda)=I+\sum_{j=1}^{\infty} R_{k}^{j} \frac{\lambda^{j}}{j !}=\exp \left\{\lambda R_{k}\right\}
$$

with the matrices $R_{k} \in \mathbb{C}^{2 n \times 2 n}$ satisfying

$$
R_{k}^{*} \mathcal{J}+\mathcal{J} R_{k}=0, \quad R_{k+N}=R_{k} .
$$

When $S$ is a symplectic matrix 'close' to the identity matrix $(\|I-S\|<1)$, then there is a well-defined matrix $\log S$, and we have

$$
S^{\lambda}=\exp \{\lambda \log S\}=\sum_{j=0}^{\infty}(\log S)^{j^{j}} \frac{\lambda^{j}}{j !}
$$

and it is known (see, e.g., [12, p.8]) that the logarithm of a symplectic matrix is the Hamiltonian matrix, i.e., $(\log S)^{*} \mathcal{J}+\mathcal{J} \log S=0$.

The symplectic system where $\mathcal{S}_{k}(\lambda)$ depend on $\lambda$ in the form (18) was investigated in [13]. Here we have

$$
S_{k}^{[j]}=\frac{R_{k}^{j}}{j !}
$$

so identity (6) holds as can be verified by a short direct computation. Moreover, it was shown in [13] that the matrix $\mathcal{A}(\lambda, \bar{\lambda})$ in (7) is of the form

$$
\mathcal{A}(\lambda, \bar{\lambda})=\sum_{j=0}^{\infty}(-1)^{j} \frac{(\bar{\lambda}-\lambda)^{2 j}}{(2 j+1) !}\left(R^{*}\right)^{j}(-\mathcal{J} R) R^{j}+\sum_{j=1}^{\infty}(-1)^{j} \frac{(\bar{\lambda}-\lambda)^{2 j-1}}{(2 j) !}\left(R^{*}\right)^{j} \mathcal{J} R^{j} .
$$

Also, [13] shows, using the time scales calculus, that the dependence of $S(\lambda)$ on $\lambda$ given by (18) is, in a certain sense, a natural discrete counterpart of the multiplicative dependence on $\lambda$ in (3).

(iv) Symplectic systems with nonlinear dependence on a spectral parameter.

In [14], oscillatory and spectral properties of (2) with real-valued $\mathcal{S}_{k}(\lambda)$ were investigated. The crucial assumption in that paper is that the matrices ( with $^{\circ}=\frac{d}{d \lambda}$ )

$$
\Psi_{k}(\lambda):=\dot{\mathcal{S}}_{k}(\lambda) \mathcal{J} \mathcal{S}_{k}^{T}(\lambda) \quad \text { are symmetric }
$$

(and nonpositive definite). We show that our assumptions (4), (5), and (6) imply (20). First of all, observe that the symplecticity of a matrix $\mathcal{S}$ is equivalent to the symplecticity of its 
transpose $\mathcal{S}^{T}$. Hence, identities (4), (5), (6) are equivalent (for real $S^{[j]}$ ) to

$$
\begin{aligned}
& S^{[1]} \mathcal{J}+\mathcal{J}\left(S^{[1]}\right)^{T}=0, \\
& S^{[2]} \mathcal{J}+\mathcal{J}\left(S^{[2]}\right)^{T}+S^{[1]} \mathcal{J}\left(S^{[1]}\right)^{T}=0, \\
& \vdots \\
& S^{[n]} \mathcal{J}+\cdots+S^{[n-j]} \mathcal{J}\left(S^{[j]}\right)^{T}+\cdots+\mathcal{J}\left(S^{[n]}\right)^{T}=0 .
\end{aligned}
$$

We have (suppressing the index $k$ )

$$
\begin{aligned}
\dot{\mathcal{S}}(\lambda) & \mathcal{J} \mathcal{S}^{T}(\lambda) \\
= & \left(\sum_{j=1}^{\infty} j \lambda^{j-1} S^{[j]}\right) \mathcal{J}\left(\sum_{j=0}^{\infty} \lambda^{j} S^{[j]}\right)^{T} \\
= & S^{[1]} \mathcal{J}+\lambda\left[S^{[1]} \mathcal{J}\left(S^{[1]}\right)^{T}+2 S^{[2]} \mathcal{J}\right]+\lambda^{2}\left[S^{[1]} \mathcal{J}\left(S^{[2]}\right)^{T}+2 S^{[2]} \mathcal{J}\left(S^{[1]}\right)^{T}+3 S^{[3]} \mathcal{J}\right]+\cdots \\
& +\lambda^{n}\left[S^{[1]} \mathcal{J}\left(S^{[n]}\right)^{T}+2 S^{[2]} \mathcal{J}\left(S^{[n-1]}\right)^{T}+\cdots+j S^{[j]} \mathcal{J}\left(S^{[n+1-j]}\right)^{T}+\cdots\right. \\
& \left.+(n+1) S^{[n+1]} \mathcal{J}\right]+\cdots .
\end{aligned}
$$

We will show that the matrices by all powers of $\lambda$ are symmetric. Suppose that $n=2 j-1$ is odd; if $n$ is even, the situation is similar. We arrange the summands by $\lambda^{n}$ in brackets in the previous formula into the triangular scheme as follows:

$$
\begin{array}{r}
S^{[1]} \mathcal{J}\left(S^{[n]}\right)^{T}+\cdots+S^{[j]} \mathcal{J}\left(S^{[j]}\right)^{T}+S^{[j+1]} \mathcal{J}\left(S^{[j-1]}\right)^{T}+\cdots+S^{[n+1]} \mathcal{J} \\
\vdots \\
+S^{[j]} \mathcal{J}\left(S^{[j]}\right)^{T}+S^{[j+1]} \mathcal{J}\left(S^{[j-1]}\right)^{T}+\cdots+S^{[n+1]} \mathcal{J} \\
+S^{[j+1]} \mathcal{J}\left(S^{[j-1]}\right)^{T}+\cdots+S^{[n+1]} \mathcal{J} \\
\vdots \\
+S^{[n+1]} \mathcal{J} .
\end{array}
$$

Substituting for the first $j$ lines in this expression from (21) (with $n+1$ instead of $n$ ), we can write (22) as (we arrange again the first $j$ lines into a triangular-type scheme)

$$
\begin{aligned}
& -\mathcal{J}\left(S^{[n+1]}\right)^{T} \\
& -\mathcal{J}\left(S^{[n+1]}\right)^{T}-S^{[1]} \mathcal{J}\left(S^{[n]}\right)^{T} \\
& \vdots \\
& -\mathcal{J}\left(S^{[n+1]}\right)^{T}-S^{[1]} \mathcal{J}\left(S^{[n]}\right)^{T}-\cdots-S^{[j-1]} \mathcal{J}\left(S^{[j+1]}\right)^{T} \\
& \quad+S^{[j+1]} \mathcal{J}\left(S^{[j-1]}\right)^{T}+\cdots+S^{[n]} \mathcal{J}\left(S^{[1]}\right)^{T}+S^{[n+1]} \mathcal{J}
\end{aligned}
$$




$$
\begin{aligned}
& +S^{[n]} \mathcal{J}\left(S^{[1]}\right)^{T}+S^{[n+1]} \mathcal{J} \\
& +S^{[n+1]} \mathcal{J}
\end{aligned}
$$

Now we see that the sum of the terms on the first and the last line is a symmetric matrix (since $\mathcal{J}^{T}=-\mathcal{J}$ ). Similarly, the sum of the second and last but one line terms is a symmetric matrix, etc. Altogether, the term by $\lambda^{n}, n \in \mathbb{N}$, is a symmetric matrix, which means that the matrix $\Psi$ is really symmetric.

\section{Central stability zone}

We rewrite the matrices $\mathcal{S}(\lambda)$ in system (2) into the form

$$
\mathcal{S}_{k}(\lambda)=I+\lambda S_{k}^{[1]}+o(\lambda) \quad \text { as } \lambda \rightarrow 0
$$

with $S^{[1]}$ satisfying (4). The monodromy matrix of (2) is

$$
\mathcal{U}_{N}(\lambda):=\mathcal{S}_{N-1}(\lambda) \cdots \mathcal{S}_{0}(\lambda)=I+\lambda \sum_{k=0}^{N-1} S_{k}^{[1]}+o(\lambda)
$$

as $\lambda \rightarrow 0$. Denote

$$
\mathcal{S}^{[1]}=\sum_{k=0}^{N-1} S_{k}^{[1]} .
$$

Then, for $\lambda \in \mathbb{R}$ with $|\lambda|$ small, we have $\log \mathcal{U}_{N}(\lambda)=\lambda \mathcal{S}^{[1]}+o(\lambda)$. If we denote by $\gamma_{j}(\lambda)$ the eigenvalues of the matrix $\log \mathcal{U}_{N}(\lambda)$, we have

$$
\gamma_{j}(\lambda)=\lambda s_{j}+o(\lambda)
$$

where $s_{j}$ are the eigenvalues of $\mathcal{S}^{[1]}$.

The proof of the following statement (which shows the non-emptiness of the central stability zone under an additional assumption that the eigenvalues of a certain matrix are distinct) is essentially the same as that of [2, Theorem 2.2].

Theorem 1 Suppose that the matrix $\mathcal{J} \mathcal{S}^{[1]}$ is negative definite and the eigenvalues $s_{j}$ of $\mathcal{S}^{[1]}$ are distinct. Then there exists $l>0$ such that the solutions of (2) are bounded on $\mathbb{Z}$ for real $\lambda$ satisfying $|\lambda|<l$.

Proof The proof is based on the statement that if $A$ is a Hermitean positive definite matrix, then the equation $\operatorname{det}(A+i \mu \mathcal{J})=0$ has only real roots; see [15]. So, let $A=-\mathcal{J S}^{[1]}$ and look for the eigenvalues of $\mathcal{S}^{[1]}$ in the form $i \sigma$. Then

$$
0=\operatorname{det}\left(\mathcal{S}^{[1]}-i \sigma I\right)=\operatorname{det} \mathcal{J}\left(-\mathcal{J} \mathcal{S}^{[1]}+i \sigma \mathcal{J}\right)=\operatorname{det}(A+i \sigma \mathcal{J})
$$

(since $\operatorname{det} \mathcal{J}=1$, see [1]), and hence $\sigma \in \mathbb{R}$ and $s_{j}=i \sigma_{j}$. Now, for $\lambda \in \mathbb{R}$, $|\lambda|$ small, the numbers $\gamma_{j}(\lambda)=i \sigma_{j} \lambda+o(\lambda)$ are still different. However, at the same time, for $\lambda \in \mathbb{R}$, the matrix $\mathcal{U}_{N}(\lambda)$ is symplectic, i.e., $\mathcal{U}_{N}^{*}(\lambda) \mathcal{J U}_{N}(\lambda)=\mathcal{J}$, and hence its eigenvalues $\rho_{j}(\lambda)=\exp \left\{\gamma_{j}(\lambda)\right\}$ 
are symmetric with respect to the unit circle, and hence $\gamma_{j}(\lambda)$ are symmetric with respect to the imaginary axis. But this implies that $\gamma_{j}(\lambda)$ themselves are on the imaginary axis, i.e., $\left|\rho_{j}(\lambda)\right|=1$. This means, in view of the later given formula (25), that solutions of (2) are bounded on $\mathbb{R}$ for $\lambda \in \mathbb{R},|\lambda|$ small.

\section{Krein's traffic rules}

So far we did not need any assumption on the matrices $S(\lambda)$ for $\lambda \in \mathbb{C}$. Complex values of $\lambda$ are needed to get an extension of the previous theorem to the case when the eigenvalues of $\mathcal{S}^{[1]}$ are not necessarily distinct. In this situation, the stability behavior of solutions of (2) is heavily based on Krein's traffic rules for the eigenvalues of the monodromy matrix $\mathcal{U}_{N}(\lambda)$.

The crucial concept is the concept of a positive-type system. In the Hamiltonian setting (14), this means

$$
H_{k} \geq 0, \quad k=0, \ldots, N-1 \quad \text { and } \quad \sum_{k=0}^{N-1} H_{k}>0
$$

see [7], where

$$
H_{k}=\left(\begin{array}{cc}
-C_{k} & A_{k}^{*} \\
A_{k} & B_{k}
\end{array}\right)
$$

here $>(\geq,<, \leq)$ means positive (nonnegative, negative, nonpositive) definiteness of the matrix indicated. Under this assumption, we have for solutions $z=\left(\begin{array}{l}x \\ u\end{array}\right)$ of system (14)

$$
\Delta\left(z_{k}^{*} \mathcal{J} z_{k}\right)=(\bar{\lambda}-\lambda)\left(\begin{array}{c}
x_{k+1} \\
u_{k}
\end{array}\right)^{*} H_{k}\left(\begin{array}{c}
x_{k+1} \\
u_{k}
\end{array}\right) \geq 0
$$

see [7]. Consequently, one can speak about $\mathcal{J}$-monotonicity of the monodromy matrix $\mathcal{U}_{N}(\lambda)$ depending on whether $\operatorname{Im} \lambda>0$ or $\operatorname{Im} \lambda<0$, in the same way as in [2].

In our symplectic setting, we have

$$
S_{k}^{*}(\lambda) \mathcal{J} S_{k}(\lambda)-\mathcal{J}=(\bar{\lambda}-\lambda)\left[-\mathcal{J} S_{k}^{[1]}+G_{k}(\lambda, \bar{\lambda})\right]
$$

where $G_{k}$ is the matrix containing powers of $\lambda$ and $\bar{\lambda}$ such that

$$
G_{k}(\lambda, \bar{\lambda})=\mathcal{J} S_{k}^{[1]}+\mathcal{A}_{k}(\lambda, \bar{\lambda})
$$

see (7). Actually, as we will see later (proof of Theorem 2), the eigenvalues of the monodromy matrix (i.e., multipliers) follow Krein's traffic rules if we suppose that there exists $\varepsilon>0$ such that

$$
\mathcal{A}_{k}(\lambda, \bar{\lambda}) \geq 0, \quad k=0, \ldots, N-1 \quad \text { and } \quad \sum_{k=0}^{N-1} \mathcal{A}_{k}(\lambda, \bar{\lambda})>0 \quad \text { for }|\operatorname{Im} \lambda|<\varepsilon
$$

A closer examination of the proofs in [2] reveals that when investigating the behavior of eigenvalues of the monodromy matrix $\mathcal{U}_{N}(\lambda)$ in dependence on the parameter $\lambda$, one 
can forget about the original system from which this monodromy matrix originates (differential system (3) or difference system (2)). The only important fact is that this matrix is symplectic for $\lambda \in \mathbb{R}$ and $\mathcal{J}$-monotonic for $\lambda \in \mathbb{C} \backslash \mathbb{R}$. Under assumption (24), the traffic rules of eigenvalues of $\mathcal{U}_{N}(\lambda)$ are the same as in $[2,6]$.

Recall now some important facts and concepts. Let $\xi \in \mathbb{C}^{2 n}$ be an eigenvector of $\mathcal{U}_{N}(\lambda)$ corresponding to the eigenvalue $\rho$, which is called the multiplier of $\mathcal{U}_{N}(\lambda)$. The solution $z$ of (2) given by the initial condition $z_{0}=\xi$ satisfies

$$
z_{N}=\mathcal{U}_{N}(\lambda) \xi=\rho \xi=\rho z_{0},
$$

and generally $z_{k+N}=\rho z_{k}$. Consequently, solutions of (2) are bounded for $k \in \mathbb{Z}$ if and only if all eigenvalues of $\mathcal{U}_{N}(\lambda)$ have modulus one and are of simple type, i.e., multiple eigenvalues have the same algebraic and geometric multiplicity.

Clearly, $\lambda=0$ is the stability point of (2) since all solutions are constant in this case. Theorem 1 states that if the eigenvalues of $\mathcal{S}^{[1]}$ are distinct, we have a central stability zone for (2). The restriction on distinctness of eigenvalues is removed in [2] (see also [6, 7] in the discrete case) using a perturbation principle, then Theorem 1 remains true when Krein's traffic rules for eigenvalues are preserved. For these rules, the crucial concept is the concept of a multiplier of definite type which is defined as follows.

Definition 1 Let $\left|\rho_{0}\right|=1$ be an eigenvalue of the monodromy matrix $\mathcal{U}_{N}$, and let $\mathcal{L}_{\rho_{0}}$ be its eigenspace. If $i u^{\prime \prime} \mathcal{J} u>0$ for every $u \in \mathcal{L}_{\rho_{0}}$, then the eigenvalue $\rho_{0}$ is said to be the multiplier of the first kind and it is called of second kind if $i u^{*} \mathcal{J} u<0$ for $u \in \mathcal{L}_{\rho_{0}}$. If there exists $0 \neq u \in \mathcal{L}_{\rho_{0}}$ such that $u^{*} \mathcal{J} u=0$, the multiplier $\rho_{0}$ is of indefinite (mixed) type. If $\left|\rho_{0}\right| \neq 1$ is an eigenvalue of $\mathcal{U}_{N}$, then it is called of the first kind if $\left|\rho_{0}\right|>1$ and of the second kind if $\left|\rho_{0}\right|<1$.

The following statement is proved similarly as [6, Theorem 4.6].

Theorem 2 Suppose that (24) holds and that for $\lambda_{0} \in \mathbb{R}$ the monodromy matrix $\mathcal{U}_{N}\left(\lambda_{0}\right)$ has a multiplier $\rho_{0}$, with $\left|\rho_{0}\right|=1$, of definite kind with the multiplicity $m>0$. Then in a $\delta$-neighborhood of $\lambda_{0}, m$ eigenvalues $\rho_{1}(\lambda), \ldots, \rho_{m}(\lambda)$ of $\mathcal{U}_{N}(\lambda)$, for which $\rho_{j}(\lambda) \rightarrow \rho_{0}$ as $\lambda \rightarrow \lambda_{0}, j=1, \ldots, m$, are situated. Moreover, if $\lambda \in \mathcal{O}_{\delta}\left(\lambda_{0}\right)$ is real, then $\rho_{j}(\lambda), j=1, \ldots, m$, stay on the unit circle. The coefficients $\alpha_{j}$ in the expansion

$$
\rho_{j}(\lambda)=\rho_{0}\left[1+\alpha_{j}\left(\lambda-\lambda_{0}\right)+o\left(\lambda-\lambda_{0}\right)\right]
$$

are given by

$$
\alpha_{j}=-\frac{1}{u_{j}^{*} \mathcal{J} u_{j}} \sum_{k=0}^{N-1}\left(z_{k}^{[j]}\right)^{*}\left[-\mathcal{J} S_{k}^{[1]}+G_{k}(\lambda, \bar{\lambda})\right] z_{k}^{[j]},
$$

where $u_{j}$ are properly taken eigenvectors of $\mathcal{U}_{N}\left(\lambda_{0}\right)$ corresponding to $\rho_{0}$ and $z_{k}^{[j]}=\mathcal{U}_{k}\left(\lambda_{0}\right) u_{j}$ are the solutions of (2) with $\lambda=\lambda_{0}$ given by the initial condition $z_{0}^{[j]}=u_{j}$. 
Proof The only difference with respect to the proof of Theorem [6, Theorem 4.6] is that for $\lambda \in \mathcal{O}_{\delta}\left(\lambda_{0}\right)$ and solutions $z^{[j]}, j=1, \ldots, m$, we have

$$
\begin{aligned}
\left(z_{N}^{[j]}\right)^{*} \mathcal{J} z_{N}^{[j]}-u_{j}^{*} \mathcal{J} u_{j} & =\sum_{k=0}^{N-1}\left(z_{k}^{[j]}\right)^{*}\left[S_{k}^{*}(\lambda) \mathcal{J} S_{k}(\lambda)-\mathcal{J}\right] z_{k}^{[j]} \\
& =-2 i \operatorname{Im} \lambda \sum_{k=0}^{N-1}\left(z_{k}^{[j]}\right)^{*}\left[-\mathcal{J} S_{k}^{[1]}+G_{k}(\lambda, \bar{\lambda})\right] z_{k}^{[j]} .
\end{aligned}
$$

Hence

$$
\left(\left|\rho_{j}(\lambda)\right|^{2}-1\right) u_{j}^{*} \mathcal{J} u_{j}=-2 i \operatorname{Im} \lambda \sum_{k=0}^{N-1}\left(z_{k}^{[j]}\right)^{*}\left[-\mathcal{J} S_{k}^{[1]}+G_{k}(\lambda, \bar{\lambda})\right] z_{k}^{[j]}
$$

and for $\lambda=\lambda_{0}+i h, h \in \mathbb{R}$, similarly as in $[2,6]$,

$$
\lim _{h \rightarrow 0} \frac{1}{2 i h}\left(1-\left|\rho_{j}\left(\lambda_{0}+i h\right)\right|^{2}\right)=-\frac{\rho_{j}^{\prime}\left(\lambda_{0}\right)}{\rho_{j}\left(\lambda_{0}\right)}=-\frac{\rho_{j}^{\prime}\left(\lambda_{0}\right)}{\rho_{0}} .
$$

The previous parts of our paper show that if (2) is of definite type, i.e., (24) holds, then we have

$$
-\left.i \frac{\rho_{j}^{\prime}(\lambda)}{\rho_{j}(\lambda)}\right|_{\lambda=\lambda_{0}}=\left.\frac{d}{d \lambda} \arg \rho(\lambda)\right|_{\lambda=\lambda_{0}}<0
$$

for the first kind multipliers and $\frac{d}{d \lambda} \arg \rho(\lambda)>0$ for the second kind ones. This means that the multipliers resulting from splitting of first kind multipliers move clockwise and second kind multipliers move counterclockwise with increasing $\lambda \in \mathbb{R}$, both remain on the unit circle in some neighborhood of $\lambda_{0}$.

Now, let us take $\lambda_{0}=0$, which is surely the stability point. Then, under assumptions of Theorem 1, system (2) is stable in some neighborhood of $\lambda_{0}=0$. The multipliers move around the unit circle as described above and may exit the unit circle only when multipliers of different kind meet on the unit circle. However, this may happen only when one of the multipliers comes through the point $[-1,0]$ on the unit circle, i.e., $\rho(\lambda)=-1$ is the eigenvalue of $\mathcal{U}_{N}(\lambda)$, which is the same as that the boundary value problem

$$
z_{k+1}=S_{k}(\lambda) z_{k}, \quad z_{N}+z_{0}=0
$$

has a solution.

Here we have, similarly to [7], the different situation in comparison with [6], where the symplectic system corresponding to the second-order system (12) is considered; see the part (i) of Section 2. Since in our case $\mathcal{U}_{N}(\lambda)$ is no longer a polynomial, generally it can happen that the equation $\operatorname{det}\left(\mathcal{U}_{N}(\lambda)+I\right)=0$ has no root, and hence (2) is stable for every $\lambda$.

Let us comment shortly on assumption (24). In the Hamiltonian setting treated in the part (ii) of Section 2, we have

$$
\mathcal{A}_{k}(\bar{\lambda}, \lambda)=D_{k}^{*}(\lambda) H_{k} D_{k}(\lambda)
$$


where $H$ is given by (23) and $D(\lambda)$ by (17). Hence, (24) is satisfied for all $\lambda \in \mathbb{C}$, not only for those with $|\operatorname{Im} \lambda|$ sufficiently small. Concerning the exponential case of Section 2(iii), we have (compare (19))

$$
\mathcal{A}_{k}(\bar{\lambda}, \lambda)=-\mathcal{J} R_{k}+o(\operatorname{Im} \lambda) \quad \text { as } \operatorname{Im} \lambda \rightarrow 0 .
$$

Hence, (24) is satisfied if

$$
-\mathcal{J} R_{k} \geq 0, \quad k=0, \ldots, N-1 \quad \text { and } \quad-\sum_{k=0}^{N-1} \mathcal{J} R_{k}>0 .
$$

This condition is in agreement with assumptions on the matrices $H_{k}$ in the Hamiltonian setting. In the general setting, (24) is satisfied if

$$
-\mathcal{J} S_{k}^{[1]} \geq 0, \quad k=0, \ldots, N-1, \quad-\sum_{k=0}^{N-1} \mathcal{J} S_{k}^{[1]}>0
$$

and

$$
G_{k}(\bar{\lambda}, \lambda)=o(\operatorname{Im} \lambda) \quad \text { as } \operatorname{Im} \lambda \rightarrow 0, k=0, \ldots, N-1
$$

Finally, note that we have made only the first step towards the elaboration of a consistent stability theory of periodic symplectic difference systems in our paper. For example, we have not been able yet to establish a 'symplectic version' of the perturbation principle which enables to prove the statement of Theorem 1 without the additional assumption that the eigenvalues of the matrix $\mathcal{S}^{[1]}$ are distinct. This is one of the problems which are subject of the present investigation, and we hope to solve it, together with other open problems, in subsequent papers.

\section{Competing interests}

The author declares that they have no competing interests.

\section{Acknowledgements}

The author thanks referees (especially one of them) for valuable remarks and suggestions which substantially contributed to the present version of the paper. The research is supported by the grant 201/10/1032 of the Czech Grant Agency (GAČR).

Received: 20 December 2012 Accepted: 11 March 2013 Published: 2 April 2013

\section{References}

1. Dopicio, FM, Johnson, CR: Complementary bases in symplectic matrices and a proof that their determinant is one. Linear Algebra Appl. 419, 772-778 (2006)

2. Krein, MG: The basic propositions of the theory of $\lambda$-zones of stability of a system of linear differential equations with periodic coefficients. In: In Memory of Aleksandr Aleksandrovič Andronov, pp. 413-498. Izdat. Akad. Nauk. SSSR, Moscow (1955) (Russian). English translation: Four papers on ordinary differential equations. Edited by Lev J. Leifman. Transl. Am. Math. Soc., Ser. 2 120, 1-70 (1983). Am. Math. Soc., Providence

3. Bohner, M, Došlý, O, Kratz, W: Sturmian and spectral theory for discrete symplectic systems. Trans. Am. Math. Soc. 361, 3109-3123 (2009)

4. Kratz, W: Discrete oscillation. J. Differ. Equ. Appl. 9, 135-147 (2003). In honour of Professor Allan Peterson on the occasion of his 60 th birthday, Part II

5. Kelley, WG, Peterson, AC: Difference Equations: An Introduction with Applications. Academic Press, San Diego (2001)

6. Halanay, A, Rasvan, V: Stability and BVP's for discrete-time linear Hamiltonian systems. Dyn. Syst. Appl. 9, 439-459 (1999)

7. Rasvan, V: Stability zones for discrete time Hamiltonian systems. Arch. Math. 36, 563-573 (2000)

8. Gelfand, IM, Lidskii, VB: On the structure of regions of stability of linear canonical systems with periodic coefficients. Usp. Mat. Nauk 10(1(63)), 3-40 (1955) (Russian). English translation: Transl. Am. Math. Soc. 8, 143-181 (1958) 
9. Krein, MG, Yakubovich, VA: Hamiltonian systems of linear differential equations with periodic coefficients. In: Analytic Methods in the Theory of Non-Linear Vibrations (Proc. Internat. Sympos. Non-linear Vibrations, vol. I, 1961), pp. 277-305. Izdat. Akad. Nauk Ukrain. SSR, Kiev (1963) (Russian, English summary)

10. Yakubovich, VA, Starzhinskii, VM: Linear Differential Equations with Periodic Coefficients, vols. 1, 2. Israel Program for Scientific Translations, Jerusalem (1975)

11. Rasvan, V: On the stability zones for discrete-time periodic linear Hamiltonian systems. Adv. Differ. Equ. 2006, Article ID 80757 (2006)

12. Ekeland, I: Convexity Methods in Hamiltonian Mechanics. Springer, Berlin (1990)

13. Došlý, O: Symplectic difference systems: natural dependence on a parameter. Adv. Differ. Syst. Appl. (to appear)

14. Hilscher, RŠ: Oscillation theorems for discrete symplectic with nonlinear dependence in spectral parameter. Linear Algebra Appl. 437, 2922-2960 (2012)

15. Gantmacher, FR: The Theory of Matrices, vols. 1, 2. AMS Chelsea Publishing, Providence (1998)

doi:10.1186/1687-1847-2013-85

Cite this article as: Došlý: Symplectic difference systems with periodic coefficients: Krein's traffic rules for multipliers. Advances in Difference Equations 2013 2013:85.

\section{Submit your manuscript to a SpringerOpen ${ }^{\ominus}$ journal and benefit from:}

- Convenient online submission

- Rigorous peer review

- Immediate publication on acceptance

Open access: articles freely available online

- High visibility within the field

- Retaining the copyright to your article 